\title{
Regional metabolism: associations with dyscalculia in Alzheimer's disease
}

\author{
Nobutsugu Hirono, Etsuro Mori, Kazunari Ishii, Toru Imamura, Tatsuo Shimomura, \\ Satoshi Tanimukai, Hiroaki Kazui, Mamoru Hashimoto, Hikari Yamashita, \\ Masahiro Sasaki
}

Division of Clinical

Neurosciences

N Hirono

E Mori

T Imamura

T Shimomura

S Tanimukai

$\mathrm{H}$ Kazui

M Hashimoto

H Yamashita

Division of

Neuroimaging

Research, Hyogo

Institute for Aging

Brain and Cognitive

Disorders, Himeji,

Japan

K Ishii

M Sasaki

Correspondence to: Dr Nobutsugu Hirono,

Division of Clinical

Neuroscience, Hyogo

Institute for Aging Brain and

Cognitive Disorders, 520

Saisho-ko, Himeji 670-0981,

Japan. Telephone 0081792

955511 ; fax 008179295

8199; email

hirono@hiabcd.go.jp

Received 5 March 1998 and in revised form

29 May 1998

Accepted 15 June 1998

\begin{abstract}
Objectives-The ability to calculate, which is an important aspect of social daily living, is commonly impaired in patients with Alzheimer's disease even early in the course of the disease. Dyscalculia is often accompanied by focal brain damage, and has been argued to be an independent sign localised around the left temporoparietal region. However, the region most responsible for dyscalculia in Alzheimer's disease has not been determined. The relation between calculation ability and regional cerebral glucose metabolism in Alzheimer's disease was therefore examined.

Methods-The calculation ability, In 91 patients with probable Alzheimer's disease of minimal to moderate severity, was assessed using the arithmetic subtest of the Wechsler adult intelligence scalerevised and the performance correlated with regional cerebral glucose metabolism determined by ${ }^{18} \mathrm{~F}$-fluorodeoxyglucose and PET.

Results-Regional glucose metabolism in the left inferior parietal lobule and in the left inferior temporal gyrus was significantly correlated with the calculation performance irrespective of age, sex, education, and severity of disease.

Conclusions-The results suggest that dysfunction of the left inferior parietal lobule and the left inferior temporal gyrus plays an important part in producing dyscalculia in patients with Alzheimer's disease.

(F Neurol Neurosurg Psychiatry 1998;65:913-916)

Keywords: calculation; Alzheimer's disease; left inferior parietal lobule
\end{abstract}

The ability to calculate is important for many aspects of social daily living, especially in shopping, financing, and business affairs. Dyscalculia is often accompanied by focal brain damage, and has been argued to be an independent sign localised around the left temporoparietal region. ${ }^{1-3}$ Impairment of calculation ability is also a common symptom in patients with Alzheimer's disease, which can appear early in the course of the disease. ${ }^{3-5}$ However, the pathological basis that is most relevant to dyscalculia in Alzheimer's disease is still unclear. The purpose of this study was to determine whether there are any anatomical correlates of dyscalculia by using PET of regional cerebral glucose metabolism (CMRglc).

\section{Methods}

According to the following criteria, 91 Japanese patients with Alzheimer's disease were selected from those who were given a short term admission to our hospital for examination between February 1994 and October 1997. After a complete description of the study was given to the subjects and their relatives, written informed consent was obtained. The inclusion criteria were (1) the NINCDS/ADRDA criteria for probable Alzheimer's disease, ${ }^{6}$ and (2) minimal to moderate functional severity. The exclusion criteria were (1) complications of other neurological diseases or illness, (2) any evidence of focal brain lesions on MRI and of cerebral arterial occlusive lesions on MR angiography, (3) the presence of severe aphasia, severe cognitive, attentional, or behavioural disorders that would make assessment of calculation and PET examination difficult $(\geqslant 6$ on the digit span subtest of the Wechsler adult intelligence scale-revised (WAIS-R), ${ }^{7} \geqslant 8$ on the repetition subscale, and $\geqslant 6$ on the comprehension subscale of the western aphasia battery $\left.(\mathrm{WAB})^{8}\right)$, and (4) left handed or ambidextrous.

The mean (SD) age of the patients was 69.3 (SD 8.2) years and the mean educational level was 9.2 (SD 2.3) years. There were 65 women and 26 men. Functional severity as rated by the clinical dementia rating scale $(\mathrm{CDR})^{9}$ was 0.5 (minimal) in 16 patients, 1 (mild) in 59 patients, and 2 (moderate) in 16 patients. The mean mini mental state examination $(\mathrm{MMSE})^{10}$ score was 19.6 (SD 4.1).

A semiquantitative assessment of calculation was done by using the arithmetic subtest of the WAIS- $\mathrm{R}^{7}$ and the calculation subtest of the WAB. $^{8}$ The WAIS-R arithmetic subtest consists of 18 arithmetical story problems of increasing difficulty. The subjects were asked to answer within time limits ranging from $15 \mathrm{sec}-$ onds on the first four problems to 60 seconds on the last nine problems. The subjects were not allowed to use paper or pencil. Raw score 
Partial correlation coefficients after controlling for age, sex, education, and CDR between the WAIS-R arithmetic test and normalised regional cerebral glucose metabolism

\begin{tabular}{lrr}
\hline Region & Left side & Right side \\
\hline Superior frontal gyrus & 0.118 & -0.022 \\
Middle frontal gyrus & 0.149 & 0.135 \\
Inferior frontal gyrus & 0.271 & 0.141 \\
Basal frontal cortex & -0.054 & -0.050 \\
Anterior cingulate gyrus & 0.047 & -0.097 \\
Posterior cingulate gyrus & 0.217 & 0.168 \\
Superior temporal gyrus & 0.147 & -0.008 \\
Middle temporal gyrus & 0.263 & 0.121 \\
Inferior temporal gyrus & $0.350^{\star}$ & 0.216 \\
Medial temporal region & -0.084 & -0.050 \\
Superior parietal lobule & 0.324 & 0.301 \\
Inferior parietal lobule & $0.429^{\star}$ & 0.302 \\
Lateral occipital cortex & 0.120 & 0.079 \\
Medial occipital cortex & 0.069 & 0.105 \\
Lenticular nucleus & -0.042 & -0.145 \\
Thalamus & -0.091 & -0.153 \\
\hline
\end{tabular}

${ }^{\star} \mathrm{p}<0.00156$

bonus points were given for particularly rapid responses on the last nine items. The maximum score of the test was 27. The raw score was subjected to the analysis. The normative performance of elderly people determined in our institute was 13.9 (SD 4.1). The WAB calculation subtest consists of 12 mental arithmetic problems that are presented in written forms. The subjects were requested to calculate mentally and either answer orally or choose the correct answer from four alternatives. The subjects were not allowed to use paper or pencil. Two points were given for each correct answer. The normative performance of elderly people determined in our institute was the maximum score $(=24)$.

The CMRglc was measured by PET and ${ }^{18} \mathrm{~F}$ fluorodeoxyglucose within 1 month after the neuropsychological testing as described previously, ${ }^{11}$ with the patient under resting conditions with eyes closed and ears unplugged. Two to four circular regions of interest of $10 \mathrm{~mm}$ diameter were placed on 32 cerebral regions (table). A senior neuroradiologist blinded to the patients' status was employed in region of interest placement and measurements. To increase the reliability, the values of CMRglc in each region were averaged. To remove the between subject difference in baseline metabolism, analyses were based on normalised values of regional CMRglc (nCMRglc) - that is, on the ratio of the regional CMRglc to the mean value of metabolic rates for

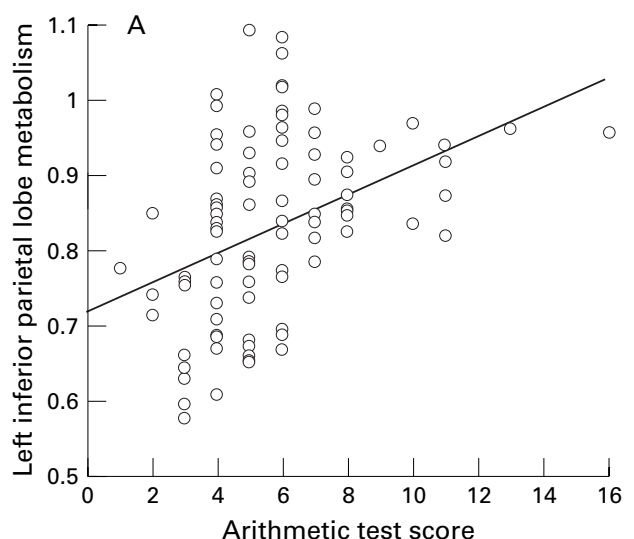

glucose in the bilateral primary sensorimotor cortices, where the histological changes are largely unaffected in Alzheimer's disease.

A Pearson correlation coefficient was initially used to analyse the relation between $\mathrm{nCMRglc}$ of each brain region and each calculation test score. Subsequently, a multiple linear regression partial correlation was calculated with each test score as a dependent variable and $\mathrm{nCMRglc}$ of each brain region as an independent variable, to eliminate the possible effect of age, sex, education, and CDR, as these variables were incorporated in the model. Because 32 repeated comparisons were involved for each calculation test, we adopted a conservative $\alpha$ level of $0.05 / 32=0.00156$. All statistical analyses were carried out on SAS release 6.10 (SAS Institute Inc, Cary, NC, USA).

\section{Results}

The mean score of the WAIS- $\mathrm{R}$ arithmetic test was 5.7 (SD 2.5). The Pearson correlation analyses showed that the test scores correlated significantly with nCMRglc in the left inferior parietal lobule $(r=0.405, \mathrm{p}<0.0001)$ and in the left inferior temporal gyrus $(r=0.381$, $\mathrm{p}=0.0002)$. Even after controlling for the effects of age, sex, educational attainment, and CDR in the multiple regression analyses, these correlations remained significant (table 1). No other significant region was noted. Scatter plots illustrate the relations between the arithmetic test score and nCMRglc in both regions (figure). The mean score of the WAB calculation test was 22.7 (SD 2.5). Because more than two thirds of the patients $(n=65)$ achieved the full score on this test, it was not further analysed.

\section{Discussion}

In the present study, dyscalculia in patients with minimal to moderate Alzheimer's disease was evident in the performance of the WAIS-R arithmetic story problem test but less perceptible in the performance on the WAB calculation problem test. The WAIS-R story problems assess the knowledge of and the ability to apply arithmetical operations without testing symbol recognition or spatial dyscalculia. On the other hand, the performance on the WAB calculation

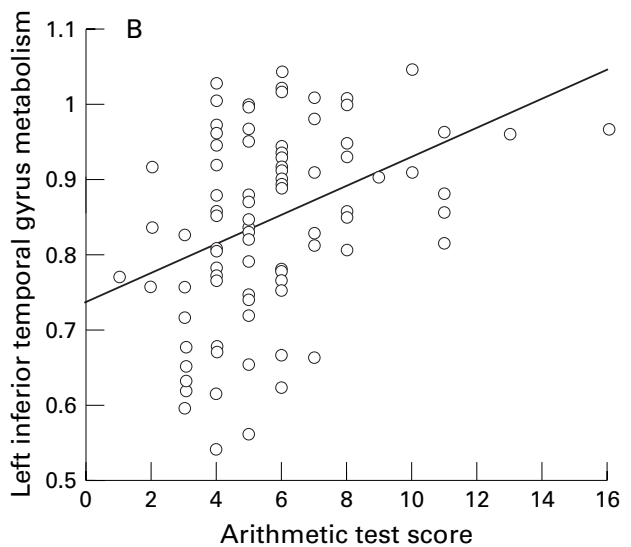

Relation between the WAIS-R arithmetic test score and normalised cerebral glucose metabolism in (A) the left inferior parietal lobule $(r=0.405, p<0.0001)$ and $(B)$ in the left inferior temporal gyrus $(r=0.381, p=0.0002)$. 
subtest is strongly and adversely affected by an inability to recognise symbols and spatial dyscalculia as well as by poor arithmetical skills. ${ }^{12}$ Our results indicated that in patients with minimal to moderate Alzheimer's disease, knowledge and skill of arithmetic operations are the main features of the dyscalculia, whereas symbol recognition disability and spatial dyscalculia are largely insignificant attributes. Attentional, language, and memory impairments may affect the test performance. However, in the present study, as patients with attentional and language impairments severe enough to interfere with the performance of the tests were not included, involvement of these factors is unlikely to be significant. Although verbal short term memory is a prerequisite for maintenance of arithmetical stories, sentence repetition was well preserved in all patients.

The main finding of the present study is that dyscalculia in Alzheimer's disease was significantly correlated with glucose hypometabolism in the left inferior parietal lobule and in the left inferior temporal gyrus. Only a few studies have explored the relation between dyscalculia and regional brain dysfunction in patients with Alzheimer's disease and they have varying methodological limitations. These include spurious correlations caused by severity of dementia that should be factored out, the lack of statistical power with small sample size, and inappropriate application of statistical analysis including multiple comparisons. Previous studies using single photon emission computed tomography (SPECT) or PET have shown conflicting results; they have shown a correlation with hypoperfusion in the left posterior temporal and parietal regions, ${ }^{13}$ with hypoperfusion in the left parieto-occipital region, ${ }^{14}$ or with hypoperfusion in the right anterior inferior frontal and anterior superior parietal regions,${ }^{15}$ or have failed to show correlations with any specific regions. ${ }^{16}$ However, based on many studies of patients with focal brain damage, dyscalculia has been localised to lesions in the left temporoparietal region including the left inferior parietal lobule, ${ }^{34}$ although there is no specific region uniquely underlying calculation impairment. ${ }^{17}$ The role of the posterior parietal cortex in arithmetical calculation was also shown by activation studies in normal subjects. ${ }^{18}{ }^{19}$ Roland et al, ${ }^{18}$ measuring regional cerebral blood flow with the intracarotid ${ }^{133} \mathrm{Xe}$ injection technique, found that the blood flow increased in the bilateral angular cortices during a silent serial subtraction task. Rueckert et $a l,{ }^{19}$ by using functional MRI, showed a significant cortical activation in bilateral posterior parietal cortices as well as premotor and prefrontal cortices during a silent serial subtraction task. Our findings that dyscalculia in patients with Alzheimer's disease is attributable to dysfunction of the inferior parietal lobule is consistent with those findings of dyscalculia in patients with focal brain damage and those in brain activation studies in normal subjects.

A peculiar finding in this study is that the glucose metabolism in the left inferior temporal gyrus was associated with the performance on the WAIS-R story problems. Little direct evidence is available from lesion studies and functional neuroimaging studies indicating a role of this region in calculation. The left inferior temporal gyrus is involved in semantic memory processing. ${ }^{20}$ Therefore, the association between the left inferior temporal function and the calculation performance is likely attributable to this region's function, which subserves the knowledge of arithmetical operations that are essential for solving arithmetical story problems. Kennedy et $a l^{1}$ reported a pedigree with familial Alzheimer's disease, in which neuropsychological profiles were characterised by an initial memory deficit and early dyscalculia. In those patients, severe dyscalculia was evident on the WAIS-R arithmetic subtest, and glucose hypometabolism in the left inferior temporal gyrus was prominent. Their findings are consistent with the results of our correlational analysis.

In conclusion, the results of the present study suggest that damage to the left inferior parietal lobule, where dyscalculia has commonly been localised by studies of patients with focal brain damage and by functional MRI studies, also plays an important part in impairing calculation ability in patients with Alzheimer's disease. The present regional metabolic study successfully demonstrated the functional neuroanatomy of calculation impairment in Alzheimer's disease. This result shows, in agreement with a previous study, ${ }^{22}$ how studies of brain-behaviour relations in patients with Alzheimer's disease may be useful for determining the cortical localisation of subtle cognitive functions.

We thank Dr Hajime Kitagaki (Division of Neuroimaging Research) for his help in various parts of the study, and Yoko Takatsuki and Akitsugu Tokimasa (Neurorehabilitation Service) for their technical assistance. This study was supported by the Medical Research Fund of Hyogo Medical Association (1997)

1 Grafman J, Passafiume D, Faglioni P, et al. Calculation disturbances in adults with focal hemispheric damage. Cortex 1982;18:37-50.

2 Boller F, Grafman J. Acalculia: historical development and current significance. Brain Cogn 1983;2:205-23.

3 Deloche G, Hannequin D, Carlomagno S, et al. Calculation and number processing in mild Alzheimer's disease. $7 \mathrm{Clin}$ Exp Neuropsychol 1995;17:634-9.

4 Grafman J, Kampen D, Rosenberg J, et al. The progressive breakdown of number processing and calculation ability: a case study. Cortex 1989;25:121-33.

5 Parlato V, Lopez OL, Panisset M, et al. Mental calculation in mild Alzheimer's disease: a pilot study. Int $f$ Geriatr Psychiatry 1992;7:599-602

6 McKhann G, Drachman D, Folstein M, et al. Clinical diagnosis of Alzheimer's disease: report of the NINCDSADRDA Work Group under the auspices of Department of Health and Human Services Task Force on Alzheimer's Disease. Neurology 1984;34:939-44.

7 Wechsler D. Manual for the Wechsler adult intelligence scale-revised. New York: Psychological Corporation, 1981.

8 Kertesz A. The western aphasia battery. New York: Grune and Stratton, 1982.

9 Hughes CP, Berg L, Danziger WL, et al. A new clinical scale for the staging of dementia. Br F Psychiatry 1982;140:56672

10 Folstein MF, Folstein SE, McHugh PR. Mini-mental state: a practical method for grading the cognitive state of patients for the clinician. F Psychiatr Res 1975;12:189-98.

1 Ishii K, Sasaki M, Kitagaki H, et al. Reduction of cerebellar glucose metabolism in advanced Alzheimer's disease. $\mathcal{F}$ Nucl Med 1997;38:925-8.

12 Lezak MD. Neuropsychological assessment, 3rd ed. New York: Oxford University Press, 1995.

13 Hunter R, McLuskie R, Wyper D, et al. The pattern of function-related regional cerebral blood flow investigated by single photon emission tomography with ${ }^{99 \mathrm{~mm}} \mathrm{Tc}$ HMPAO in patients with presenile Alzheimer's disease and Korsakoff's psychosis. Psychol Med 1989;19:847-55

14 Engel P, Cummings JL, Villanueva-Meyer J, et al. Single photon emission computed tomography in dementia: 
relationship of perfusion to cognitive deficits. $\mathcal{F}$ Geriatr Psychiatry Neurol 1993;6:144-51

15 Osimani A, Ichise M, Chung DG, et al SPECT for differential diagnosis of dementia and correlation of rCBF with cognitive impairment. Can f Neurol Sci 1994;21:104-11.

16 Nybäck H, Nyman H, Blomqvist G, et al. Brain metabolism in Alzheimer's dementia: studies of ${ }^{11} \mathrm{C}$-deoxyglucose accumulation, CSF monoamine metabolites and neuropsychological test performance in patients and healthy subjects. $\mathscr{F}$ Neurol Neurosurg Psychiatry 1991;54:672-8.

17 Kahn HJ, Whitaker HA. Acalculia: an historical review of localization. Brain Cogn 1991;17:102-15.

18 Roland PE, Friberg L. Localization of cortical areas activated by thinking. $\mathcal{F}$ Neurophysiol 1985;53:1219-43.
19 Rueckert L, Lange N, Partiot A, et al. Visualizing cortical activation during mental calculation with functional MRI. Neuroimage 1996;3:97-103.

20 Hodges JR. Disorders of semantic memory. $\mathcal{F}$ Neurol Neurosurg Psychiatry 1997;62:431-5.

21 Kennedy AM, Newman SK, Frackowiak RSJ, et al. Chromosome 14 linked familial Alzheimer's disease. A clinico-pathological study of a single pedigree. Brain 1995; 118:185-205.

22 Penniello MJ, Lambert J, Eustache F, et al. A PET study of the functional neuroanatomy of writing impairment in Alzheimer's disease. The role of the left supramarginal and left angular gyri. Brain 1995;118:697-706.

\section{NEUROLOGICAL STAMP}

\section{Jean Baptiste van Helmont (1577-1644)}

In the fune issue of the fournal this stamp was inadvertently published with the wrong vignette. Here it appears again with the correct one.

Jean Baptiste van Helmont was the founder of the latrochemical School, which looked to chemical explanations of vital phenomena. He was a man of great intellectual curiosity, who studied philosophy at Louvain. Disappointed with the content of the study he turned to law and after further disappointment decided to study medicine.

Van Helmont's teaching revolved around two words, "Blas" and "Gas". He was much influenced by the doctrines of Paracelsus and, like Paracelsus, thought that each material process of the body was presided over by a special spirit. This he called Blas. Physiological processes were themselves chemical, being activated by a special ferment (Gas) and were also presided over by a special spirit. This spirit was supposedly governed by a sensory-motor sensitive soul, the very principle of life, situated in the folds of the stomach, especially its orifice. It was thought that here the duumvirate of stomach and spleen regulated the functions of life and here epilepsy was engendered. When the duumvirate "withdraws its government" epilepsies or other diseases resulted. Van Helmont conceded that the "occasional nest" of epilepsy could be in the head or feet. Although in his opinion the disease originated in the stomach he considered that it could be provoked by strong emotions affecting the sensitive soul.

There was another disease which van Helmont thought related to epilepsy. This was asthma. It had its original seat in the duumvirate. It affected and shook the whole body, but then concentrated on the lungs-whereas epilepsy made itself felt in the head. Van Helmont was so impressed by the apparent analogy between the two diseases that he stated: "We may lawfully, therefore, by a Phylosophical Liberty name an asthma the falling sickness of the lungs".

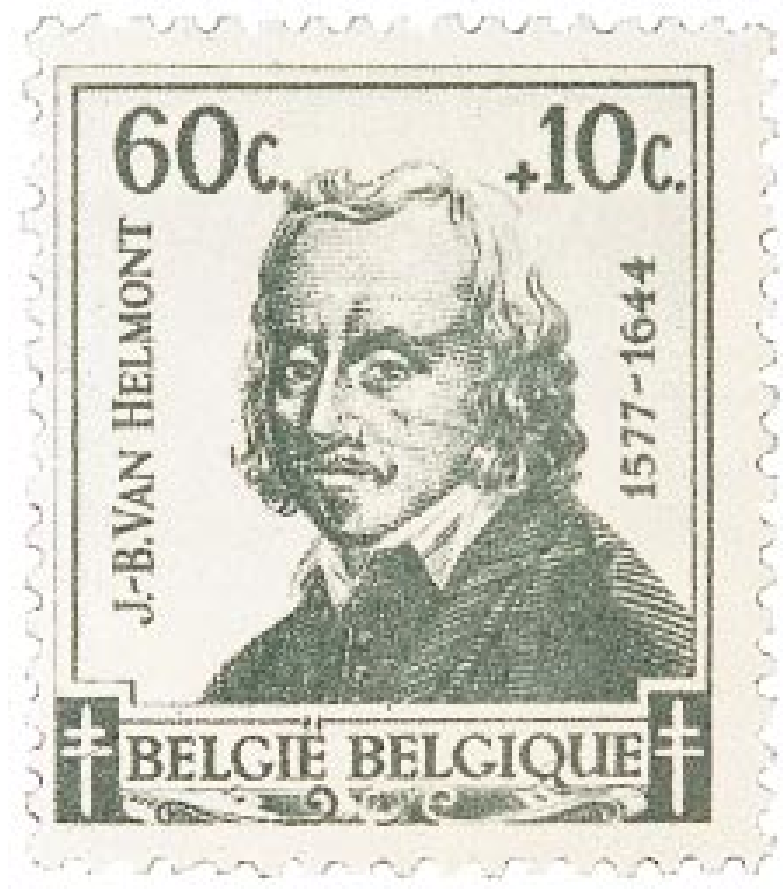

Van Helmont believed in Paracelsus' weapon salve, which healed by anointing the weapon instead of the wound and he became involved in a controversy regarding the weapon salve and magnetic and sympathetic healing. In 1624 the Inquisition denounced his views and declared him suspect of heresy. His chief work, Ortus Medicinae, was published in 1648, after his death. Van Helmont was honoured philatelically on a Belgium stamp issued in 1942 (Stanley Gibbons 989, Scott B322)

L F HAAS 